\title{
Condução do Tratamento com Hormônio de Crescimento (GH) nos Pacientes com Diagnóstico de Deficiência GH (DGH) Durante o Período de Transição da Criança para o Adulto
}

revisão

\author{
EVANDRo S. Portes
}

ERIKA BARBOSA

Serviço de Endocrinologia e Metabologia do Hospital do Servidor Estadual de São Paulo,

SP, Brasil.

\author{
RESUMO
}

Além de estimular o crescimento estatural, o hormônio de crescimento (GH) promove outros efeitos benéficos nos pacientes com deficiência de GH (DGH). A suspensão do $\mathrm{GH}$ em pacientes com $\mathrm{DGH}$, durante o período de transição da criança para a vida adulta, induz a alterações metabólicas desfavoráveis na composição corporal, na integridade óssea, na capacidade para desempenhar atividade física, e também aumenta fatores de risco cardiovasculares. Estes parâmetros melhoram quando a reposição do GH é reiniciada em adultos com DGH. Com base nestas evidências, a reposição do $\mathrm{GH}$ não deveria ser suspensa quando o paciente atingisse sua altura final e, sim, mantida durante a vida adulta. Entretanto, considerando que muitos pacientes com diagnóstico de DGH, quando criança, não tem este diagnóstico confirmado no início da vida adulta, é necessário reavaliar a secreção de $\mathrm{GH}$ quando o paciente atingir a altura final. A história clínica do paciente, a resposta ao tratamento com GH, a ressonância magnética da região hipotalâmicahipofisária e a concentração de IGF-1 podem ajudar nesta reavaliação. A realização de testes de estímulo para liberação do GH é necessária, a menos que o paciente apresente lesão estrutural ou genética que justifiquem a deficiência deste hormônio. (Arq Bras Endocrinol Metab 2008; 52/5:854-860)

Descritores: Hormônio do crescimento; Hormônio do crescimento/deficiência; Hormônio do crescimento/uso terapêutico; Composição corporal.

\section{ABSTRACT}

Management of the Growth Hormone (GH)-Treated Patients with Diagnosis of GH Deficiency (DGH) During Transition from Childhood to Adulthood.

Growth hormone $(\mathrm{GH})$ has many beneficial effects in patients with childhoodonset GH deficiency (GHD) in addition to its promotion of linear growth. The discontinuation of GH treatment in GHD patients, during the transition from childhood to adulthood, induces significant unfavorable changes in body composition, skeletal integrity, exercise capacity, and an adverse cardiovascular risk profile. These changes are reversed after the resumption of $\mathrm{GH}$ treatment. As the benefits of continuing GH therapy into adulthood has been well established, it is possible that GH replacement therapy will not be stopped once growth has been completed, but it will continue into adult life. Considering that a high proportion of patients with diagnosis of DGH in childhood are no longer GHD in adolescence, the GH status must be retested when growth is completed. Other factors such as clinical history, GH response in childhood, hipotalamic-pituitary MRI and IGF-1 concentration must be considered. Reconfirmation of GHD diagnosis through stimulation testing is usually required, unless there is a proven genetic or structural lesion persistent from childhood. (Arq Bras Endocrinol Metab 2008; 52/5:854-860)

Recebido em $1 / 6 / 2008$ Aceito em 8/6/2008
Keywords: Growth hormone; Growth hormone/therapeutic use; Growth hormone/deficiency; Body composition. 


\section{INTRODUÇÃo}

$\mathrm{O}$ TRATAMENTO COM hormônio de crescimento $(\mathrm{GH})$ tem sido utilizado desde o final dos anos 1950 (1), com a finalidade de promover o aumento da estatura final em crianças e adolescentes, principalmente nos pacientes com diagnóstico da deficiência de GH (DGH). Até o início dos anos 1980 este tratamento era realizado com GH extraído da hipófise de cadáver humano, sendo a sua produção escassa. O uso desta medicação era direcionado quase exclusivamente às crianças com quadro clínico sugestivo de deficiência mais grave de GH. O tratamento era suspenso tão logo o paciente fechasse, ou chegasse perto do fechamento das cartilagens de crescimento. O foco deste tratamento era apenas o crescimento estatural. Somente após 1985, com o início da produção do GH pela técnica do DNA recombinante, houve maior disponibilidade da medicação para todas as crianças que apresentavam baixa estatura secundária à DGH. A partir dessa época, diversos centros de pesquisa passaram a avaliar o tratamento com $\mathrm{GH}$ em crianças com diagnóstico de baixa estatura sem critérios clássicos de DGH, e também em adultos com diagnóstico de deficiência deste hormônio.

O grande desafio, durante estes quase 50 anos, tem sido identificar corretamente os pacientes portadores desta deficiência e que, portanto, poderiam realmente se beneficiar com a sua reposição. Para iniciar a investigação da DGH em crianças e adolescentes, deve-se valorizar, além da história clínica, os dados auxológicos. Somente pacientes com alta probabilidade de serem portadores dessa deficiência deveriam prosseguir em investigação. Para diagnosticar esta patologia, pode-se dosar o GH, o fator de crescimento semelhante à insulina (IGF-1) e a proteína ligadora de IGF-1 tipo 3 (IGFBP3) no sangue, e o GH na urina. Além destes exames, a imagem da região hipotalâmica hipofisária obtida pela ressonância magnética $(\mathrm{RM})$ e o estudo molecular do gene do $\mathrm{GH}$, dos genes responsáveis pela sua transcrição em nível hipofisário e de outros genes envolvidos na sua produção e secreção auxiliam no estabelecimento do correto diagnóstico. Nos adultos, este diagnóstico é mais difícil, pois com a finalização do crescimento estatural, os dados auxológicos passam a não ter importância como ferramenta diagnóstica.

A dosagem do GH após testes de estímulo são pouco reprodutíveis, eles apresentam grande variabilidade de respostas, além de serem testes farmacológicos que nem sempre expressam a liberação fisiológica deste hor- mônio (2). Todavia, a dosagem sérica do $\mathrm{GH}$, em diversas amostras colhidas dentro do intervalo de 12 ou 24 horas, assim como sua dosagem na urina, não se mostraram superiores a estes testes de estímulo na avaliação de pacientes com suspeita de DGH (3). A dosagem do IGF-1 e da IGFBP3, apesar de agregarem novas informações, auxiliando no diagnóstico da criança com baixa estatura, também estão longe de ser exames considerados padrão-ouro para este diagnóstico. $\mathrm{O}$ avanço nos exames de imagem, principalmente da RM, tem contribuído para o diagnóstico mais preciso desta patologia. $\mathrm{O}$ estudo genético tem evoluído muito nos últimos anos, possibilitando o diagnóstico inequívoco desta deficiência. Porém, atualmente o estudo molecular identifica somente pequena parcela dos pacientes que tem nos defeitos moleculares o fator etiológico desta patologia.

Ainda hoje, o objetivo principal do tratamento com GH em crianças e adolescentes é a promoção do crescimento estatural. Entretanto, após o início da utilização do GH recombinante, houve grande aumento nos trabalhos que avaliaram o impacto da reposição deste hormônio no organismo humano como um todo. Ficou demonstrado que, além de promover o crescimento estatural, a reposição de $\mathrm{GH}$ altera a composição corporal, aumentando a massa muscular, a massa óssea e o volume líquido extracelular, e diminui o percentual do tecido adiposo, os fatores de risco cardiovasculares, a morbidade e talvez a mortalidade associada a esta patologia, além de melhorar a qualidade de vida destes pacientes $(4,5)$. Estes parâmetros pioram, no paciente com diagnóstico de $\mathrm{DGH}$, quando o GH é suspenso ao final da fase de crescimento estatural, e melhoram quando a reposição de $\mathrm{GH}$ é iniciada em adultos com este diagnóstico $(4,6)$. Estas evidências são bem observadas no paciente com $\mathrm{DGH}$, no período de transição da adolescência para a vida adulta, por ocasião da interrupção do GH. Este período é caracterizado como o tempo decorrido do final da puberdade até seis a sete anos após o paciente atingir sua estatura final (7). Em 1996, fundamentado nestas evidências, a Food and Drug Administration (FDA) liberou o uso do GH para o tratamento de pacientes adultos com diagnóstico de DGH.

\section{MANIFESTAÇÕES CLÍNICAS E METABÓLICAS}

As principais manifestações clínicas e metabólicas da deficiência de GH nesta fase de transição são: 


\section{Composição corporal}

A presença do GH é necessária para o ganho de massa e força muscular no período de transição da adolescência para a vida adulta (8). A massa e a força muscular aumentam gradativamente após o fechamento das cartilagens de crescimento, porquanto o pico de massa muscular ocorre entre os 20 e 30 anos de idade (8). A suspensão do tratamento com GH, quando o paciente com DGH atinge a estatura final, reduz a massa e a força muscular $(5,8-10)$, comprometendo a capacidade para a atividade física (11). Estas alterações são reversíveis com a reposição de $\mathrm{GH}(5,12)$.

A deficiência de GH aumenta o tecido adiposo, tanto em nível subcutâneo quanto em nível visceral (4-6,9). A reposição deste hormônio aumenta a lipólise, principalmente da gordura visceral $(2,12)$, diminuindo o percentual de tecido adiposo nos portadores de DGH (12). Ao suspender esta reposição, ocorre novamente o aumento do tecido adiposo corporal $(5,9)$.

\section{Massa óssea}

A densidade mineral óssea continua a aumentar após o fechamento das cartilagens de crescimento (13). O pico da massa óssea ocorre dentro do período de transição e é dependente da ação do GH (7,14-16). A suspensão da reposição de $\mathrm{GH}$ em adolescentes com DGH leva à diminuição dos marcadores de formação e reabsorção óssea, indicando a redução na atividade de remodelação óssea $(13,14)$. A reposição de $\mathrm{GH}$ melhora a densidade mineral óssea tanto em jovens quanto em adultos com DGH (16-18). Entretanto, o ganho de massa óssea observado nos pacientes em tratamento com GH durante o período de transição é muito maior que o observado em pacientes portadores de DGH após esta faixa etária. Parece existir uma janela de oportunidade na qual a reposição de GH seja essencial. Por conseguinte, pacientes com DGH devem manter a reposição de $\mathrm{GH}$, mesmo após terem atingido sua estatura final, para que alcancem maior densidade mineral óssea $(4,15,16,19)$. A dose de $\mathrm{GH}$ preconizada para obter o ganho adequado de massa óssea durante este período é menor que a comumente utilizada em crianças e adolescentes. A dose mais elevada de GH durante esta fase poderia levar à desincronização do turnover ósseo, fazendo que a reabsorção prevaleça sobre a formação óssea. Isso explicaria o me- nor ganho de massa óssea observado em pacientes tratados com doses de GH comumente utilizadas em adolescentes quando comparados aos pacientes tratados com doses utilizadas em adultos (16).

\section{Risco cardiovascular}

A deficiência de GH está associada ao aumento dos fatores de risco para doenças cardiovasculares, o que poderia contribuir para o aumento da mortalidade nos pacientes portadores de $\mathrm{DGH}$, reduzindo a expectativa de vida nesta população (5). A reposição de $\mathrm{GH}$ contribui para normalizar o tamanho do coração, que está reduzido nos portadores de $\mathrm{DGH}$, e para diminuir os níveis séricos de colesterol (20), o que poderia contribuir para a aceleração do processo aterosclerótico neste grupo de pacientes. Contudo, recentemente foi demonstrado que a $\mathrm{DGH}$ isolada não induz ao aumento da aterosclerose (21). Embora existam referências do aumento de mortalidade por causas cardiovasculares nos pacientes com DGH (22), estes dados devem ser mais bem avaliados pois em muitos pacientes a concomitância de outras deficiências hormonais, como a de T4 e a dos esteróides sexuais, assim como a reposição de quantidade elevada de cortisol, ou o tratamento radioterápico, podem ser causa de o aumento da mortalidade nestes pacientes (23).

Com as claras evidências que a reposição do GH exerce funções fisiológicas muito importantes no paciente portador de DGH, e com a relativa facilidade para sua obtenção, apesar de o alto custo financeiro, surgiram algumas questões: 1) Deve-se manter a reposição de GH durante a vida adulta em todos os pacientes com diagnóstico de DGH na infância e na adolescência? 2) Este tratamento deve ser mantido com base em exames realizados por ocasião do diagnóstico inicial ou seria necessária a reavaliação da secreção de GH nestes pacientes?

Diversos autores demonstraram que pacientes que tiveram diagnóstico de DGH quando crianças ou adolescentes, tendo como parâmetro a ausência de resposta do $\mathrm{GH}$ aos testes de estímulo farmacológico, quando submetidos a novo teste para liberação de $\mathrm{GH}$ ao término do tratamento, já no final da adolescência ou quando adultos jovens passam a apresentar resposta normal de secreção do $\mathrm{GH}$ a estes testes, em um percentual que varia de $12 \%$ a $81 \%$, de acordo com o grupo de pacientes estudados (24-29). Esta grande variação percentual de recuperação da secreção de GH observada neste grupo de pacientes, previamente diagnostica- 
dos como portadores de DGH, provavelmente decorre da falta de critérios uniformes para o diagnóstico da DGH, assim como a grande heterogeneidade dos pacientes nos diversos estudos realizados.

$\mathrm{O}$ diagnóstico de $\mathrm{DGH}$, realizado em crianças e adolescentes, deve, portanto, ser confirmado quando a criança atingir sua estatura final ou chegar perto dela. A reavaliação do paciente com diagnóstico de DGH deve ser realizada após suspensão da aplicação do $\mathrm{GH}$ por período de pelo menos 30 dias $(2,7,30)$. O teste considerado padrão-ouro para reavaliar a secreção de GH nestes pacientes é o teste de tolerância à insulina (ITT), porém, ele é contra-indicado em pacientes convulsivos ou portadores de cardiopatias (31). Outros testes, como o da arginina, o GHRH, o glucagon, e mesmo o da clonidina, podem ser realizados durante esta fase da vida. Apesar de a recomendação de alguns autores para não realizar o teste da clonidina na investigação de DGH em adultos, pela baixa probabilidade de induzir a liberação de $\mathrm{GH}$ nestes pacientes $(4,7,31)$, adolescentes e mesmo adultos jovens podem ainda responder bem a este estímulo, e, quando isso acontece, o diagnóstico é realizado de maneira simples, prática e segura. $\mathrm{O}$ teste da clonidina pode ser realizado como primeira escolha, e somente quando não houver resposta a este estímulo se consideraria a possibilidade de usar o ITT ou o GHRH + arginina, para confirmação diagnóstica. Em adultos mais idosos o teste do GHRH + arginina tem mostrado sensibilidade e especificidade semelhantes ao ITT $(6,32,33)$. Vários fatores influenciam o padrão de resposta do $\mathrm{GH}$ aos testes de estímulo, entre eles: estímulo empregado, idade, percentual de gordura corporal, presença de esteróides sexuais em quantidade adequada para a faixa etária, deficiências hipofisárias associadas e o tratamento adequado destas $(34,35)$.

Um consenso, publicado em 1998, definiu como critério para o diagnóstico de deficiência de GH no adulto a incapacidade de o paciente elevar a concentração plasmática de GH para mais que $3 \mu \mathrm{g} / \mathrm{L}$ após hipoglicemia induzida por insulina (ITT) (31). Um dos trabalhos que serviu como embasamento para esta diretriz avaliou a resposta do $\mathrm{GH}$ a testes de estímulo em pacientes na faixa etária de 45 anos (36). Este valor de referência foi extrapolado por muitos anos para outras faixas etárias, e utilizado na interpretação da resposta do $\mathrm{GH}$ aos testes de estímulo para liberação de $\mathrm{GH}$, em adolescentes no final do crescimento estatural, e também em adultos jovens. Entretanto, em um grupo de 26 pacientes com idade média de aproximadamente 20 anos, e com alta probabilidade de realmente terem DGH pelo fato de a maioria deles ter mais do que uma deficiência de hormônio hipofisário, ou por apresentarem alteração anatômica da região hipotalâmica-hipofisária, os níveis de resposta foram superiores a $3 \mu \mathrm{g} / \mathrm{L}$ em $23 \%$ desta amostra estudada e maiores que $5 \mu \mathrm{g} / \mathrm{L}$ em $11 \%$, sugerindo realmente que deve-se ter um nível de corte mais elevado para pacientes no final da adolescência e mesmo nos primeiros anos da idade adulta (37). Por conseguinte, aplicar em adolescentes no final do seu crescimento estatural, ou em adultos jovens, a mesma interpretação da resposta do GH aos testes de estímulo, que é utilizada em adultos após a quarta década de vida, talvez não seja o mais adequado. De acordo com o critério que for estabelecido, será alterado o percentual de pacientes com suficiência ou insuficiência de GH. Esta constatação levou a Sociedade Européia de Endocrinologia Pediátrica a estabelecer para pacientes no final da adolescência e em adultos jovens um valor de corte mais alto para se caracterizar a secreção normal de GH. Atualmente, o valor de corte recomendado por esta sociedade é de $5 \mu \mathrm{g} / \mathrm{L}$ (7). Usando o valor de corte de 5,1 $\mu \mathrm{g} / \mathrm{L}$ no teste do ITT e $4,1 \mu \mathrm{g} / \mathrm{L}$ no teste do GHRH + arginina, Biller e cols. (32) obtiveram sensibilidade e especificidade para diagnosticar pacientes com DGH, acima de $95 \%$ e $91 \%$, respectivamente.

O nível sérico de IGF-1 somente deve ser valorizado quando ajustado para o sexo, o grau de desenvolvimento puberal e a idade óssea do paciente. A sua dosagem não mostrou sensibilidade e especificidade maior que o teste de estímulo com $\mathrm{GH}$ em uma população de adultos jovens (37). Níveis normais de IGF-1 não excluem DGH $(2,31)$. Níveis baixos podem sugerir DGH quando são excluídas outras causas para a diminuição da IGF-1, como desnutrição, doença hepática, hipotireoidismo e doenças crônicas debilitantes (2). Apesar de a secreção normal de GH, após o estímulo farmacológico, o nível plasmático de IGF-1 pode estar abaixo dos níveis normais para a faixa etária e o sexo do paciente. Eventualmente, a secreção de GH farmacologicamente induzida não ocorre de maneira fisiológica, o que leva à diminuição do estímulo para produção de IGF-1. A definição da necessidade de reposição de GH nestes pacientes requer maiores estudos (38).

A RM da região hipotalâmica-hipofisária tem papel fundamental no diagnóstico da DGH, e deve sempre ser realizada. Vários estudos demonstraram que quando se realiza um novo teste de estímulo para liberação de $\mathrm{GH}$, no final da puberdade ou no início da vida 
adulta, o percentual de pacientes com ausência de resposta a este teste será maior entre aqueles que apresentam alterações anatômicas nesta região, identificadas pelo exame de RM $(34,39)$. Entre estas alterações, a presença da neurohipófise ectópica, adenohipófise hipoplásica ou defeitos da linha média são as mais importantes. Parece haver um contínuo na gravidade deste distúrbio, ou seja, quando a neurohipófise está localizada na eminência mediana e a haste hipofisária não é visível o paciente tem deficiência mais grave de $\mathrm{GH}$, e comumente também tem deficiência de outros hormônios hipofisários. Porém, quando a haste hipofisária é visível, mesmo que afilada, e a neurohipófise encontrase ao lado dela, esta deficiência é menos grave. Nestes casos, pode ocorrer até resposta normal do $\mathrm{GH}$ ao teste de estímulo quando o paciente é reavaliado ao final do seu crescimento estatural (34). Os pacientes que respondem normalmente ao teste de estímulo com liberação de GH, e que tem alteração anatômica nesta região, devem ser reavaliados periodicamente, talvez anualmente, pois já foi demonstrado que vários deles evoluem com DGH, ou mesmo apresentando deficiências de outros setores hipofisários com o passar do tempo (39). Os maiores índices de resposta considerada normal aos testes realizados para reavaliação da secreção de GH serão encontrados em pacientes com RM normal desta região $(24,38)$.

Para decidir sobre a manutenção da reposição de GH nos pacientes com diagnóstico de DGH que atingem a idade adulta, pode-se dividir estes pacientes em dois grupos:

\section{Pacientes com alta probabilidade de DGH}

Pacientes portadores de lesões congênitas, traumáticas ou infiltrativas em região hipotalâmica hipofisária, associadas a outras deficiências hormonais; pacientes submetidos à radioterapia nesta região; pacientes com alterações genéticas nos fatores de transcrição da hipófise, no gene do GH ou nos receptores do GHRH.

\section{Pacientes com baixa probabilidade de DGH}

Pacientes com DGH isolado, sem alteração genética conhecida e também sem alteração anatômica da região hipotalâmica hipofisária ao exame de RM.

A resposta aos testes de estímulo de liberação de GH não deve ser analisada isoladamente. Outros fatores, como história clínica, resposta ao tratamento com $\mathrm{GH}$, evolução puberal, RM da região hipotálamo-hipofisária e a dosagem de IGF-1, devem ser considerados em conjunto. Nos pacientes com alta probabilidade de DGH, níveis baixos de IGF-1 confirmam o diagnóstico, dispensando os testes de estímulo para liberação de GH neste grupo específico de pacientes $(24,30,31,40)$.

A dose preconizada de $\mathrm{GH}$ neste período não está totalmente estabelecida. A maioria dos trabalhos publicados tem utilizado uma dose bem menor que a dose normalmente utilizada em crianças e adolescentes. Quando se faz a opção de usar uma dose com base no peso corporal ela não deveria ultrapassar $12,5 \mu \mathrm{g} /$ $\mathrm{kg} /$ dia (33). Outros autores sugerem que em adultos jovens a dose inicial deveria ser de 400 e $500 \mu \mathrm{g} / \mathrm{dia}$ (2). O ajuste da dose deve ser realizado a cada 30 a 60 dias, com base nos níveis circulantes de IGF-1, aumentando a dose de 100 a $200 \mu \mathrm{g} /$ dia (2). O IGF-1 deve ser mantido no limite superior da normalidade, e reavaliado a cada seis meses, após o ajuste da dose. A dosagem de IGFBP3 não contribui neste seguimento (7). Os possíveis efeitos colaterais, tema abordado em outro artigo desta edição especial, devem ser monitorados periodicamente.

É evidente que o GH transcende sua ação sobre o crescimento estatural. Portanto, deve-se manter a reposição de $\mathrm{GH}$ em todos os pacientes com diagnóstico de DGH na infância e na adolescência que tiverem o diagnóstico confirmado quando reavaliados ao final do seu crescimento estatural.

\section{REFERÊNCIAS}

1. Raben, MS. Treatment of pituitary dwarf with human growth hormone. J Clin Endocrinol Metab. 1958;18:901-3.

2. Molitch ME, Clemmons, DR, Malozowski S, Merriam GR, Shalet SM, Vance ML. Evaluation and treatment of adult growth hormone deficiency: an endocrine society clinical practice guideline. J Clin Endocrinol Metab. 2006;91(5):1621-34.

3. Cacciari E, Tassoni P, Cicognani A, Pirazzoli P, Salardi S, Balsamo $A$, et al. Value and limits of pharmacological and physiological tests to diagnose growth hormone $(\mathrm{GH})$ deficiency and predict therapy response: first and second retesting during replacement therapy of patients defined as GH deficient. J Clin Endocrinol Metab. 1994;79:1663-9.

4. de Boer $\mathrm{H}$, van der Veen EA. Editorial: why retest young adults with childhood-onset growth hormone deficiency? J Clin Endocrinol Metab. 1997;82(7):2032-6.

5. Johannsson G, Albertsson-Wikland K, Bengtsson B. Discontinuation of growth hormone $(\mathrm{GH})$ treatment: metabolic effects in GH-deficient and GH-sufficient adolescent patients compared with control subjects. J Clin Endocrinol Metab. 1999;84(12):4516-24.

6. Vahl N, Juul A, Jørgensen JOL, Orskov H, Skakkebæk NE, Christiansen JS. Continuation of growth hormone (GH) replacement in GH-deficient patients during transition from chil- 
dhood to adulthood: a two-year placebo-controlled study. J Clin Endocrinol Metab. 2000;85(5):1874-81.

7. Clayton PE, Cuneo RC, Juul A, Monson JP, Shalet SM, Tauber $\mathrm{M}$. Consensus statement on management of the GH-treated adolescent in the transition to adult care. Eur $\mathrm{J}$ Endocrinol. 2005;152:165-70.

8. Hulthén L, Bengtsson BA, Sunnerhagen KS, Hallberg L, Grimby $\mathrm{G}$, Johannsson G. GH is needed for the maturation of muscle mass and strength in adolescents. J Clin Endocrinol Metab 2001;86(10):4765-70.

9. Rutherford OM, Jones DA, Round JM, Buchanan CR, Preece MA. Changes in skeletal muscle and body composition after discontinuation of growth hormone treatment in growth hormone deficient young adults. Clin Endocrinol (Oxf). 1991;34:469-75.

10. Carroll PV, Drake WM, Maher KT, Metcalfe K, Shaw NJ, Dunger DB, et al. Comparison of continuation or cessation of growth hormone (GH) therapy on body composition and metabolic status in adolescents with severe GH deficiency at completion of linear growth. J Clin Endocrinol Metab. 2004;89(8):3890-5.

11. Attanasio AF, Shavrikova E, Blum WF, Cromer M, Child CJ, Paskova M, et al. Continued growth hormone (GH) treatment after final height is necessary to complete somatic development in childhood-onset GH-deficient patients. J Clin Endocrinol Metab. 2004;89(10):4857-62.

12. Jorgensen JOL, Thuesen L, Müller J, Ovesen P, Skakkebaek $\mathrm{NE}$, Christiansen JS. Three years of growth hormone treatment in growth hormone deficient adults: near normalization of body composition and physical performance. Eur $\mathrm{J}$ Endocrinol. 130:224-8.

13. Drake WM, Carroll PV, Maher KT, Metcalfe KA, Camacho-Hübner, Shaw NJ, et al. The effect of cessation of growth hormone (GH) therapy on bone mineral accretion in GH-deficient adolescents at the completion of linear growth. J Clin Endocrinol Metab. 2003;88(4):1658-63.

14. Fors $H$, Bjarnason $R$, Wirén $L$, Albertsson-Wikland $K$, Bosaeus I, Bengtsson BA, et al. Currently used growth-promoting treatment of children results in normal bone mass and density. $A$ prospective trial of discontinuing growth hormone treatment in adolescents. Clin Endocrinol (Oxf). 2001;55:617-24.

15. Attanasio AF, Howell S, Bates PC, Frewer P, Chipman J, Blum WF, et al. Body composition, IGF-I and IGFBP-3 concentrations as outcome measures in severely GH-Deficient (GHD) patients after childhood GH treatment: A comparison with adult onset GHD patients. J Clin Endocrinol Metab. 2002;87(7):3368-72.

16. Shalet SM, Shavrikova E, Cromer M, Child CJ, Keller E, Zapletalová J, et al. Effect of growth hormone (GH) treatment on bone in postpubertal GH-deficient patients: a 2-year randomized, controlled, dose-ranging study. J Clin Endocrinol Metab. 2003;88(9):4124-9.

17. Saggese G, Baroncelli GI, Bertelloni S, Barsanti S. The effect of long-term growth hormone $(\mathrm{GH})$ treatment on bone mineral density in children with $\mathrm{GH}$ deficiency. Role of $\mathrm{GH}$ in the attainment of peak bone mass. J Clin Endocrinol Metab. 1996;81(8):3077-83.

18. Johannsson G, Roslin T, Bosaexjs I, Sjostrom L, Bengtsson BA. Two years of growth hormone (GH) treatment increases bone mineral content and density in hypopituitary patients with adult-onset GH deficiency. J Clin Endocrinol Metab. 1996;81(8):2865-73.

19. Baroncelli Gl, Bertelloni S, Sodini F, Saggese G. Longitudinal changes of lumbar bone mineral density (BMD) in patients with GH deficiency after discontinuation of treatment at final height; timing and peak values for lumbar BMD. Clin Endocrinol (Oxf). 2004;60:175-84.

20. Salerno MC, Esposito V, Farina V, Radetti G, Umbaldo A, Capalbo $D$, et al. Improvement of Cardiac performance and cardiovascular risk factors in children with $\mathrm{GH}$ deficiency after two years of GH replacement therapy: an observational, open, prospective, case-control study. J Clin Endocrinol Metab. 2006;91(4):1288-95.

21. Oliveira JLM, Marques-Santos C, Barreto-Filho JA, XimenesFilho R, Britto AVO, Souza AHO, et al. Lack of evidence of premature atherosclerosis in untreated severe isolated growth hormone $(\mathrm{GH})$ deficiency due to a GH-releasing hormone receptor mutation. J Clin Endocrinol Metab. 2006;91(6):2093-9.

22. Rosén T, Bengtsson BA. Premature mortality due to cardiovascular disease in hypopituitarism. Lancet. 1990;336:285-8.

23. Molitch, ME. Editorial: diagnosis of GH deficiency in adults how good do the criteria need to be? J Clin Endocrinol Metab. 2002;87(2):473-6.

24. Maghnie M, Strigazzi C, Tinelli C, Autelli M, Cisternino M, Loche S, et al. Growth hormone (GH) deficiency (GHD) of childhood onset: reassessment of GH status and evaluation of the predictive criteria for permanent GHD in young adults. J Clin Endocrinol Metab. 1999;84(2):1324-8.

25. Tauber M, Moulin P, Pienkowski C, Jouret B, Rochiccioli P. Growth hormone $(\mathrm{GH})$ retesting and auxological data in $131 \mathrm{GH}$ deficient patients after completion of treatment. J Clin Endocrinol Metab. 1997;82(2):352-6.

26. Nicolson, A, Toogood, AA Rahim A, Shalet SM. The prevalence of severe growth hormone deficiency in adults who received growth hormone replacement in childhood. Clin Endocrinol (Oxf). 1996;44(3):311-6.

27. Longobardi S, Merola B, Pivonello R, Di Rella F, Di Somma, Colao AM, et al. Reevaluation of Growth Hormone (GH) secretion in 69 adults diagnosed as $\mathrm{GH}$-deficient patients during childhood. J Clin Endocrinol Metab. 1996;81(3):1244-7.

28. Attanasio A, Howell S, Bates PC, Blum WF, Frewer P, Quigley $\mathrm{C}$, et al. Confirmation of severe $\mathrm{GH}$ deficiency after final height in patients diagnosed as $\mathrm{GH}$ deficient during childhood. Clin Endocrinol (Oxf). 2002;56:503-7.

29. Zucchini S, Pirazzoli P, Baronio F, Gennari M, Bal MO, Balsamo $A$, et al. Effect on adult height of pubertal growth hormone retesting and withdrawal of therapy in patients with previously diagnosed growth hormone deficiency. J Clin Endocrinol Metab. 2006;91(11):4271-6.

30. Growth Hormone Research Society. Consensus guidelines for the diagnosis and treatment of growth hormone $(\mathrm{GH})$ deficiency in childhood and adolescence: summary statement of the GH research society. J Clin Endocrinol Metab. 2000;85(11): 3990-3.

31. Consensus guidelines for the diagnosis and treatment of adults with growth hormone deficiency: summary statement of the growth hormone research society workshop on adult growth hormone deficiency. J Clin Endocrinol Metab. 1998; 83(2):379-81.

32. Biller BMK, Samuels MH, Zagar A, Cook DM, Arafah BM, Bonert $V$, et al. Sensitivity and specificity of six tests for the diag nosis of adult GH deficiency. J Clin Endocrinol Metab. 2002;87(5):2067-79.

33. Radovick. S, DiVall, S. Approach to the Growth Hormone-Deficient child during transition to adulthood. J Clin Endocrino Metab. 2007;92(4):1195-1200.

34. Léger J, Danner S, Simon D, Garel D, Czernichow P. Do all patients with childhood-onset growth hormone deficiency (GHD) 
and ectopic neurohypophysis have persistent GHD in adulthood? J Clin Endocrinol Metab. 2005;90(2):650-6.

35. Carel JC, Tresca JP, Letrait M, Chaussain JL, Lebouc Y, Job JC, et al. Growth hormone testing for the diagnosis of growth hormone deficiency in childhood: a population register-based study. J Clin Endocrinol Metab. 1997;82(7):2117-21.

36. Hoffman DM, O'Sullivan AJ, Baxter RC, HO KK. Diagnosis of growth-hormone deficiency in adults. Lancet. 1994;343:1064-8.

37. Maghnie M, Aimaretti G, Bellone S, Bona G, Bellone J, Baldelli $\mathrm{R}$, et al. Diagnosis of $\mathrm{GH}$ deficiency in the transition period: accuracy of insulin tolerance test and insulin-like growth factor-I measurement. Eur J Endocrinol. 2005;152:589-96.

38. Gelwane G, Garel C, Chevenne D, Armoogum P, Simon D, Czernichow $P$, et al. Subnormal serum insulin-like growth factor-i levels in young adults with childhood-onset nonacquired growth hormone $(\mathrm{GH})$ deficiency who recover normal GH secretion may indicate less severe but persistent pituitary failure. J Clin Endocrinol Metab. 2007;92(10):3788-95.
39. Di lorgi N, Secco A, Napoli F, Tinelli C, Calcagno A, Fratangeli $\mathrm{N}$, et al. Deterioration of growth hormone $(\mathrm{GH})$ response and anterior pituitary function in young adults with childhood-onset GH deficiency and ectopic posterior pituitary: a two-year prospective follow-up study. J Clin Endocrinol Metab. 2007;92(10):3875-84.

40. Aimaretti G, Baffoni C, Bellone S, Di Vito L, Corneli G, Arvat E, et al. Retesting young adults with childhood-onset growth hormone (GH) deficiency with gh-releasing-hormone-plus-arginine-test. J Clin Endocrinol Metab. 2000;85(10):3693-9.

Endereço para correspondência:

Evandro S. Portes

Av. Rouxinol 1041, cj. 304/305

04510-001 São Paulo, SP

E-mail: evandroportes@uol.com.br 